\title{
ERRATUM \\ American Physical Society \\ Proceedings of the New York Meeting
}

Through an oversight the titles of the invited papers presented at the Symposium on photoelectric and thermionic phenomena were omitted from the program of the meeting as printed in the March 15, 1930 issue (Phys. Rev. $35,656,1930)$. On page 668 the following should be inserted after abstract 45 :

Symposium on Photoelectric and Thermionic Phenomena

46. Photoelectric ionization of gases. F. L. Mohler, Bureau of Standards.

47. Photoelectric multiple ionization by x-rays. F. K. Richtmyer, Cornell University.

48. The quantum theory of the photoelectric and thermionic effects in metals. William V. Houston, California Institute of Technology.

49. The influence of surface conditions on the photoelectric effect. C. E. Mendenhall, University of Wisconsin.

50. The velocity distribution of $x$-ray photoelectrons. DR. G. Wentzel, University of Zürich. 